\title{
Learning to Model the Dynamics of Mechanical Systems with the Method of Solving Educational and Research Problems
}

\author{
Pavel P. Belonozhko ${ }^{1 *}$ and Yury V. Berchun ${ }^{1}$ \\ ${ }^{1}$ Bauman Moscow State Technical University, 2nd Baumanskaya str., 5/1, 105005, Moscow, Russia
}

\begin{abstract}
The methodological features of using software systems for modeling the dynamics of mechanical systems for solving educational research problems are considered. The traditionally established approach to studying the foundations of modeling the dynamics of technical systems is largely based on mastering the corresponding mathematical tools. With regard to modeling the dynamics of systems of solids, the mentioned approach involves, first of all, the development of skills in the preparation of ordinary differential equations (ODEs). At the same time, the formation of a mechanical calculation scheme as an «object of application of mathematics» becomes a natural stage of mathematical research, and the selection of simplifying assumptions that allow one or another idealization to correspond to a real object is carried out consciously taking into account the desire for certain internal properties of the mathematical model (for example, type and order of the ODE system). An important feature of modern systems for modeling the dynamics of solid's systems is the ability to describe the object under study directly in terms of the subject area. This feature provides an increase in the effectiveness of modeling in solving design and engineering and scientific research problems and allows you to save a qualified specialist from a laborious routine. At the same time, as experience shows, in the process of solving educational and research problems, due to the mentioned features of modern software, students have certain difficulties in mastering the fundamental conceptual base of the corresponding discipline (physics, electrical engineering, theoretical mechanics, theory of automatic control, fundamentals of computer aided design). The article gives an example of an educational research task focused on overcoming these difficulties.
\end{abstract}

\section{Introduction}

The intensive development of modern digital technologies is widely reflected in approaches to training in the field of engineering disciplines. At the same time, very dif-ferent aspects of the educational process are affected. For example, in [1], a new educational methodology, developed at the Department «Theory of Mechanisms and Machines» Bauman Moscow State Technical University, based on information and development

* Corresponding author: byelonozhko@ mail.ru 
technologies, is presented. The methodology is based on the joint use of laboratory equipment and computer 3D-models, which gives a visual represen-tation of the process of forming the curve of an involute tooth and allows to signifi-cantly increase the efficiency of the learning process. In [2] and [3], the results of scientific research carried out at Bauman Moscow State Technical University, in par-ticular, at the Department «Computer Aided Design Systems», aimed at improving the efficiency of the learning process by means of automated assessment of student metacompetencies. In [4], the results of teaching high school students the skills of independent research work at the stage of preparation for university admission are presented.

As noted, in particular, in [5], [6], [7], methodological issues related to mathematical modeling are often more difficult to understand than the models themselves and the processes studied with their help.

Academician A.N. Krylov in the famous work [8] formulated important features of applied mathematical research. As the skills needed by a qualified engineer, the worldfamous scientist-shipbuilder first of all singles out the ability to mathematically formulate an engineering problem, choose effective methods and mathematical means of solving it, and also correctly interpret the results. The thesis formulated in the cited work that mathematics for an engineer is primarily a means of solving ap-plied problems is gaining particular relevance today.

Modern packages for modeling the dynamics of solids systems allow us to describe the object under study directly in terms of the subject area. This feature provides in-creased modeling efficiency in solving engineering and research problems and allows you to save a qualified specialist from a laborious routine. At the same time, experi-ence shows that students have certain difficulties in mastering the fundamental con-ceptual base of the corresponding discipline (physics, electrical engineering, theoreti-cal mechanics, automatic control theory, the basics of computer-aided design). Mod-eling is perceived as some formal procedure, not a mathematical study.

Following [5], [6], [7], [8], [9], we single out once again the main stages of applied mathematical research:

1. The mathematical formulation of the problem.

2. The choice of research method.

3. Conducting mathematical research.

4. Analysis and interpretation of the result.

The capabilities of modern computer packages to automate the first three stages obviously imply new methodological approaches to the development of the correspond-ing mathematical tools in modern education. As the experience of the authors and their colleagues shows, a method based on solving educational research problems can be very effective. It is based on the well-known approach to conducting scientific research, based on the consideration of specially formulated model problems [10], [11], [12], which ensure the visibility of the results and facilitate their analysis. Formu-lating educational and research tasks in such a way that the purpose of the study is to better understand the conceptual framework of the discipline and the necessary mathematical apparatus, the teacher suggests the student to use both the capabilities of modern computer packages and the well-known methodological experience in conducting research.

Consider, as an example, the «Universal Mechanism» software package, which is designed to simulate the dynamics and kinematics of plane and spatial mechanical systems, represented as a system of solids, joints, and force elements [13]. The pack-age implements various methods for setting the mass-inertial characteristics of bod-ies. The mass and matrix elements of the inertia tensor of the body can be calculated according to the known geometric image and material properties, or set manually. In the second case, the researcher's capabilities are much wider, but a sufficiently deep understanding of the 
physical essence of the concepts «material point», «absolutely rigid body», «inertia tensor» is required in connection with the features of the mathe-matical description of mechanical idealizations that are compared to a real object. To acquire such an understanding, the authors proposed the use of educational re-search tasks focused on the development of the conceptual basis of theoretical me-chanics. As an example, the educational research task of a comparative analysis of various design schemes of a physical pendulum is presented below.

\section{Comparative analysis of design schemes of a physical pendulum. Selection of equivalence criteria.}

The initial design of the distributed mass pendulum shown in Fig. 1, characterized by the following parameters:

- $s_{f}=\mathrm{OC}-$ the distance from the suspension point $O$ to the center of mass of the pendulum $C$;

- $m_{f}$ - mass of the pendulum;

- $J_{\mathrm{Cf}}-$ the moment of inertia of the pendulum relative to the axis passing through the center of mass $C$, parallel to the axis of rotation. The axis of rotation passes through the suspension point $O$ perpendicular to the plane of the figure;

- $\bar{g}$ - acceleration of gravity.

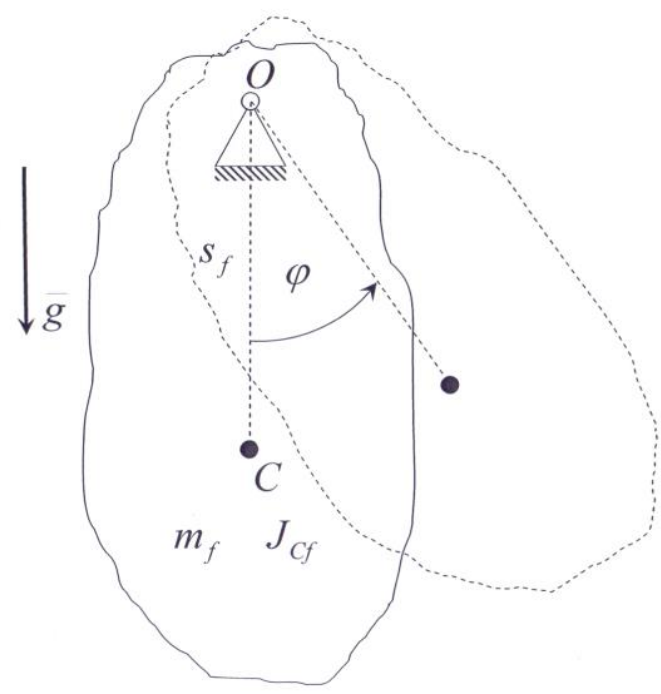

Fig. 1. Mechanical design scheme of a distributed mass pendulum.

Kinetic energy of a physical pendulum

$$
E_{\mathrm{kf}}=\frac{1}{2} J_{\mathrm{Of}} \dot{\phi}^{2}
$$

where $J_{\mathrm{Of}}=J_{\mathrm{Cf}}+m_{f} S_{f^{2}}$ - is the moment of inertia of the physical pendulum relative to the axis of rotation.

The potential energy will be assumed equal to zero in the lower position of stable equilibrium, the potential energy of the physical pendulum 


$$
E_{\mathrm{pf}}=m_{f} \mathrm{gs}_{f}(1-\cos \phi)
$$

where $g=|\bar{g}|$.

As a generalized coordinate characterizing the position of the mechanical system under consideration with one degree of freedom, we take the angle of deviation of the straight line from the vertical, counted in the positive direction counterclockwise from the lower position of stable equilibrium (Fig. 1).

We write the equation of dynamics of a physical pendulum in the form of a Lagrange equation of the second kind

$$
\frac{d}{\mathrm{dt}}\left(\frac{\partial\left(E_{\mathrm{kf}}-E_{\mathrm{pf}}\right)}{\partial \dot{\phi}}\right)-\frac{\partial\left(E_{\mathrm{kf}}-E_{\mathrm{pf}}\right)}{\partial \phi}=0 .
$$

Substituting (1) and (2) in (3), we obtain

$$
J_{\mathrm{Of}} \ddot{\phi}+m_{f} \mathrm{gs}_{f} \sin \phi=0 .
$$

Dividing (4) by $J_{\mathrm{Of}}$, we obtain

$$
\ddot{\phi}+\omega_{f^{2}} \sin \phi=0,
$$

where $\omega_{f}=\sqrt{\frac{m_{f} g_{f}}{J_{0 f}}}-$ is the natural frequency of undamped small oscillations of the physical pendulum.

Differential equation (5) is a mathematical model designed to study the law of motion $\phi(t)$ of a real object, idealized by the calculation scheme shown in Fig. 1. Regarding the considered set of properties of a real object, an equivalent model (5) will be a mathematical pendulum model of mass $m_{m}$ and length $l_{m}$

$$
\ddot{\phi}+\omega_{m^{2}} \sin \phi=0,
$$

where $\omega_{m}=\sqrt{\frac{g}{l_{m}}}-$ is the natural frequency of undamped small oscillations of the mathematical pendulum.

The reduced length of the mathematical pendulum is determined from the condition

$$
\omega_{m}=\omega_{f}
$$

$+$

$$
l_{m}=\frac{J_{\mathrm{Of}}}{m_{f} s_{f}}=\frac{J_{\mathrm{Cf}}+m_{f} s_{f}^{2}}{m_{f} s_{f}}
$$

Both mechanical calculation schemes can be easily implemented by means of the «Universal Mechanism» software package. Moreover, in the case of a mathematical pendulum, it is necessary to specify the zero matrix of the inertia tensor and some arbitrary nonzero mass.

We draw attention to the important methodological meaning of this circumstance. The concept of a "mathematical pendulum of reduced length equivalent to a physical pendulum" is well-established and involves a traditional mathematical study based on the representation of the model in the form (5) for the physical pendulum and in the form (6) for the mathematical. With this approach, the meaning of equivalence is obvious, and the independence of the parameter $\omega_{m}$ of equation (6) from the mass $m_{m}$ naturally follows from 
physical considerations that the student is forced to pay attention to in the process of obtaining the equation in form (6). Attempts to formally transfer the concept of the equivalence of a mathematical pendulum and a physical pendulum in the sense of the coincidence of equations (5) and (6) to computer models prepared by means of a package for modeling the dynamics of body systems can lead the student to the conclusion that the mass of the mathematical pendulum is a free parameter, which is set arbitrarily, while the models will be equivalent. In the absence of sufficient experience in mathematical modeling, the fallacy of this conclusion is not obvious.

An illustrative way to demonstrate the nonequivalence of computer models of mathematical and physical pendulums (in the general case of an arbitrary mass of a mathematical pendulum) is, for example, the introduction of the same small (ensuring the conservation of the oscillatory nature of the motion) dissipative articulated force in both models. In this case, the damped motion $\phi_{m}(t)$ of the mathematical pendulum, obviously, will depend on the mass $m_{m}$ and in the general case, as already noted, will not coincide with the movement $\phi_{f}(t)$ of the physical pendulum. In this case, the problem of choosing the mass $m_{m}$ can be formulated, ensuring the coincidence of damped oscillations for computer models of the mathematical and physical pendulum.

In this case, to ensure the equivalence of design schemes, it is necessary to present the mathematical pendulum model in a form similar to model (4).

Kinetic energy of a mathematical pendulum

$$
E_{\mathrm{km}}=\frac{1}{2} m_{m} l_{m^{2}} \dot{\phi}^{2} .
$$

Potential energy of a mathematical pendulum

$$
E_{\mathrm{pm}}=m_{m} \mathrm{gl}_{m}(1-\cos \phi) .
$$

The equation of dynamics of a mathematical pendulum in the form of a Lagrange equation of the second kind

$$
\begin{gathered}
\frac{d}{\mathrm{dt}}\left(\frac{\partial\left(E_{\mathrm{km}}-E_{\mathrm{pm}}\right)}{\partial \dot{\phi}}\right)-\frac{\partial\left(E_{\mathrm{km}}-E_{\mathrm{pm}}\right)}{\partial \phi}=0, \\
m_{m} l_{m^{2}} \ddot{\phi}+m_{m} \mathrm{gl}_{m} \sin \phi=0 .
\end{gathered}
$$

Equating the coefficients of equations (4) and (12) with $\ddot{\phi}$ and $\sin \phi$, we obtain

$$
\begin{gathered}
m_{m} l_{m^{2}}=J_{\mathrm{Of}}, \\
m_{m} l_{m}=m_{f} s_{f} .
\end{gathered}
$$

Dividing the first equation (13) by the second, we obtain an expression for the reduced length of the mathematical pendulum (8). Substituting (8) into the second equation (13), we obtain

$$
m_{m}=\frac{m_{f^{2} f_{f^{2}}}}{J_{\mathrm{Of}}}=\frac{m_{f^{2} s_{f^{2}}}}{J_{\mathrm{Cf}}+m_{f^{\prime}} s^{2}} .
$$

Equivalence criteria (8) and (14) ensure not only the fulfillment of condition (7), but also the fulfillment of the conditions

$$
\begin{aligned}
& E_{\mathrm{km}}=E_{\mathrm{kf}}, \\
& E_{\mathrm{pm}}=E_{\mathrm{pf}} .
\end{aligned}
$$


Thus, computer models whose parameters meet the criteria of (8) and (14) are equivalent not only in the sense of coincidence of the laws of motion, but also in the sense of the equality of the scalar fields of kinetic and potential energies determined at the points of the phase plane, which allows us to study them using not only undamped oscillations, but also movement accompanied by a change in the total energy of the system. Wherein

$$
l_{m} \neq s_{f}, m_{m} \neq m_{f}
$$

The computer models under consideration will obviously not be equivalent in the sense of a coincidence of the reaction at the suspension point (Fig. 2).
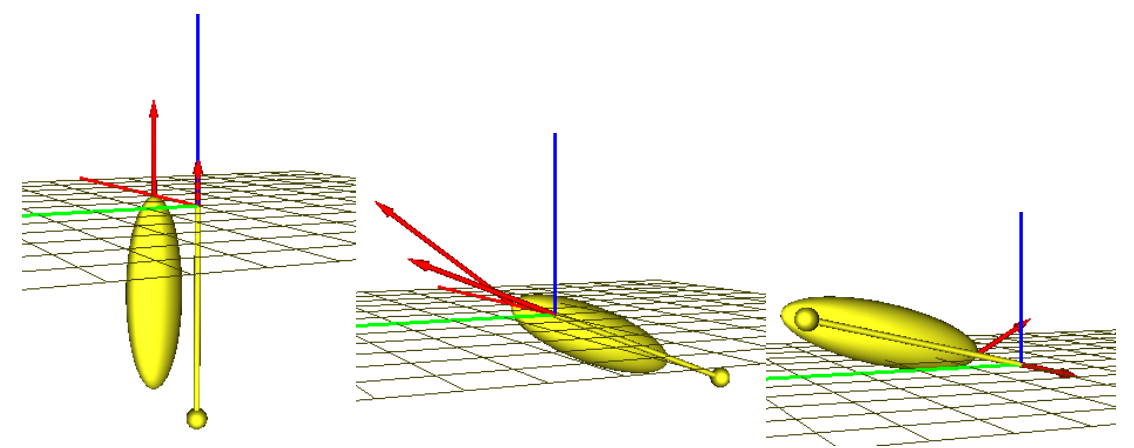

Fig. 2. The reaction force at the suspension point for computer models of mathematical and physical pendulums, equivalent in the sense of coincidence of kinetic and potential energies at each point of the phase plane.

The foregoing is easily confirmed by qualitative reasoning: in the case of a mathematical pendulum, the reaction force will always be directed along the line passing through the suspension point and the material point (along the rod). For a physical pendulum, the reaction force will have a component perpendicular to the line OC (Fig. 1), providing accelerated rotational motion of the body with a non-zero moment of inertia relative to the center of mass.

Let us now consider a system of two point masses fixed on a rectilinear weightless rod (Fig. 3).

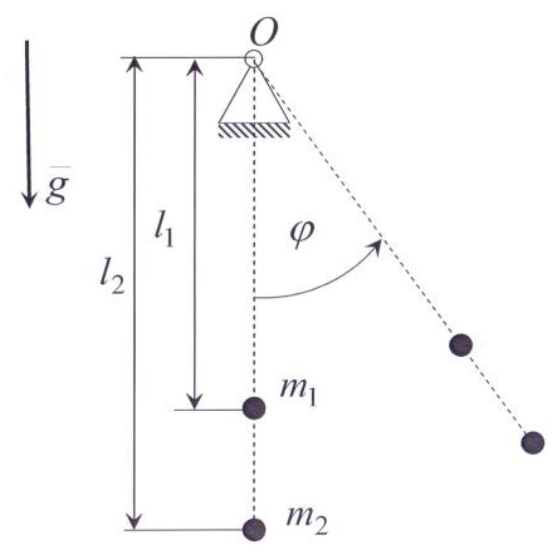

Fig. 3. Mechanical design scheme of a physical pendulum represented by two point masses 
Representing as

$$
\begin{gathered}
m_{1}=m_{2}=\frac{1}{2} m_{f}, \\
l_{1}=s_{f}-\sqrt{\frac{J_{\mathrm{Cf}}}{m_{f}}}, l_{2}=s_{f}+\sqrt{\frac{J_{\mathrm{Cf}}}{m_{f}}},
\end{gathered}
$$

we get the design scheme equivalent to the design scheme in Fig. 1 not only in the sense of fulfilling conditions (7) and (15), but also ensuring the coincidence of reactions at the suspension point. Relation (17) ensures the coincidence of the centers of mass and the equality of masses and moments of inertia of the design circuits shown in Fig. 1 and fig. 3 . A modified design diagram of Fig. Can also be considered. 3, when the mass is not concentrated at points, but distributed around a circle with a beginning at the center of mass and a radius equal to the radius of inertia of the physical pendulum $\sqrt{\frac{J_{\mathrm{Cf}}}{m_{f}}}$.

Note that all the considered schemes are easily implemented by means of modern packages for modeling the dynamics of body systems, in particular, as already noted, the «Universal Mechanism» package, and it is easy to verify the validity of the above statements about equivalence using a direct computational experiment. However, the proposed formulations of the considered equivalence criteria require the representation of mathematical models in the form of differential equations with coefficients that are functions of the mass-inertial parameters of the calculation scheme. Thus, the task of obtaining the considered criteria for equivalence can be offered to students as educational and research.

\section{Conclusion}

The given examples illustrate the methodological features of the development of software systems for modeling the dynamics of mechanical systems and possible approaches to the formulation of educational and research tasks aimed at in-depth study of the physical content of the simulated processes. It should be noted.

1. The process of choosing a meaningful model (mechanical design scheme) - a certain idealization that is mapped to a real modeling object - in a traditional mathematical study is a preliminary step in creating a mathematical model. The formal approach to this stage is fraught with obvious difficulties at the subsequent stages of the compilation and integration (research) of differential equations. The capabilities of modern computer modeling tools significantly reduce the creation of a model to the formation of a calculation scheme, which requires the formation of a responsible approach to this stage of modeling in the learning process.

2. The development of modern modeling tools often implies the parallel development of both actual computer tools and the corresponding subject area. This circumstance determines the need for new methodological approaches to learning.

3. The formation of educational research tasks, involving the use of the capabilities of modern computer packages for in-depth study of the subject area, is a promising approach to learning, allowing to increase its effectiveness. 


\section{References}

1. O. Egorova, G. Timofeev, G. Shashurin, New educational methodology to study the involute tooth profile, New Trends in Educational Activity in the Field of Mechanism and Machine Theory, Springer, Cham, pp. 29-37 (2019)

2. E.A. Gavrilina, M.A. Zakharov, A.P. Karpenko, E.V. Smirnova, A.P. Sokolov, Software System META-3 for Quantitative Evaluation of Student's Meta-competencies on the Basis of Analysis of his or her Behavior in Social Networking Services, Procedia Computer Science, 103, pp. 432-438 (2017)

3. P.P. Belonozhko, A.P. Karpenko, D.A. Khramov, Analysis of educational data: directions and prospects of application, Internet Journal of Science, 9 (4 (41)) (2017)

4. A.P. Karpenko, Features of tutoring when schoolchildren perform educational and research work in the field of engineering, BBK 72 P 23, 21.

5. A.D. Myshkis, On the teaching of mathematics to practitioners, Mathematics in Higher Education, 1, 37 (2003)

6. A.D. Myshkis, Elements of the theory of mathematical models, Ed. 3rd corrected. M.: KomKniga (2007)

7. P.V. Trusova, Introduction to Mathematical Modeling: Textbook, allowance / Ed. PV Trusova / M. M .: University book, Logos (2007)

8. A.N. Krylov, The importance of mathematics for shipbuilding, Krylov A.N. My memories.-M.: Publishing. USSR Academy of Sciences, pp. 301-314 (1963)

9. I. I. Blekhman, A.D. Myshkis, Ya.G. Panovko, Mechanics and applied mathematics, Logic and features of mathematics applications (1990)

10. P.P. Belonozhko, Proper Inertial Motion of Robotic Space Module. Reduced System Dynamics, 2018 International Conference on Industrial Engineering, Applications and Manufacturing (ICIEAM) pp. 1-5, IEEE (2018)

11. P.P. Belonozhko, Robotic Assembly and Servicing Space Module Peculiarities of Dynamic Study of Given System, Smart Electromechanical Systems, Springer, Cham, pp. 287-296 (2019)

12. P.P. Belonozhko, A Situation Control of Robotized Space Module as Multimode Dynamic Object, In Smart Electromechanical Systems, Springer, Cham, pp. 275-290 (2020)

13. «Universal mechanism». Official site. http://www.umlab.ru/ (Accessed 12.11.2019) 